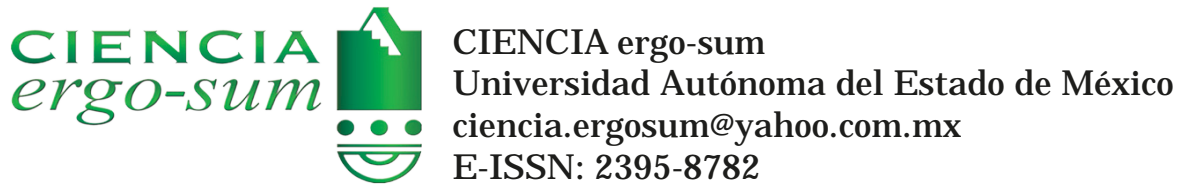

\title{
Perfiles de consumidores sin experiencia de compra en un centro comercial basados en factores de atracción: caso Cusco
}

Lodeiros Zubiria, Manuel Luis; Mauricio Andía, Martín; Munayco Abanto, Lucía; Pacheco, Milagros

Perfiles de consumidores sin experiencia de compra en un centro comercial basados en factores de atracción: caso Cusco

CIENCIA ergo-sum, vol. 26, núm. 2, julio-octubre 2019|e49

Universidad Autónoma del Estado de México, México

Esta obra está bajo una Licencia Creative Commons Atribución-NoComercial-SinDerivar 4.0 Internacional.

Lodeiros Zubiria, M. L., Mauricio Andía, M., Munayco Abanto, L. y Pacheco, M. (2019). Perfiles de consumidores sin experiencia de compra en un centro comercial basados en factores de atracción: caso Cusco. CIE NCI Aergosum, 26(2). https://doi.org/10.30878/ces.v26n2a3 


\title{
Perfiles de consumidores sin experiencia de compra en un centro comercial basados en factores de atracción: caso Cusco
}

Consumers profiles with no previous mall shopping experience based on Attractiveness Factors. Cusco's case

\author{
Manuel Luis Lodeiros Zubiria \\ Universidad Peruana de Ciencias Aplicadas, Perú \\ pccmmlod@upc.edu.pe \\ Martín Mauricio Andia \\ Universidad Peruana de Ciencias Aplicadas, Perú \\ pccmmmau@upc.edu.pe \\ Lucía Munayco Abanto \\ Universidad Peruana de Ciencias Aplicadas, Perú \\ lucia.munayco@upc.edu.pe \\ Milagros Pacheco \\ Universidad Peruana de Ciencias Aplicadas, Perú \\ u820038@upc.edu.pe
}

Recepción: 26 de abril de 2017

\section{RESUMEN}

Se busca determinar los factores que atraen a los consumidores que nunca han tenido una experiencia con un centro comercial, así como crear los perfiles de estos nuevos compradores. En una primera fase cualitativa se determinó cuáles eran los atributos de un centro comercial valorados en la realidad cusqueña. En la segunda, de orden cuantitivo, se aplicó un cuestionario a jóvenes. Con lo anterior, se identificaron los factores más influyentes a los servicios básicos y la calidad asociados a los atributos más elementales de un centro comercial. Asimismo, se definieron dos grupos de usuarios: los optimistas, quienes ven al centro comercial positivamente, aunque usan de manera limitada sus servicios, y los pesimistas, quienes muestran desidia hacia el mall.

Palabras ClaVe: centro comercial, factores de atracción, segmentación del consumidor, Cusco, comportamiento del consumidor.

\begin{abstract}
The study's goal is to determine the importance of attractiveness factors on the first shopping center in Cusco, established in 2014, and create new segments from those buyers. The research was set up in two stages. As a result of the exploration stage, the attributes concerning goods and services that adjusted to the Cusco situation were determined. In the second stage, a survey was targeted to youth people, the most important segment in Cusco. The most attractive factors were Basic Services and Quality related to the most elemental features in a shopping center. Moreover, two consumer segments were found. The first one, named as the Optimistics, who positively evaluated the mall although they used few of its services. The second group, the Pessimists, who negatively ranked the mall. KEYwORDS: Shopping Center, Attractive factors, Consumer Segmentation, Cusco, Consumer Behaviour.
\end{abstract}

\section{INTRODUCCIÓN}

Cusco es un referente turístico mundial conocido por su riqueza histórica y acervo cultural de fama universal que es capaz de atraer anualmente a millones de turistas (en 2015 fueron más de dos millones de visitantes extranjeros según datos del Instituto Nacional de Estadística e Informática-INEI). A pesar de su prestigio como punto de atracción turística, y contrario a lo que se pudiera pensar, la ciudad no se ha convertido en un polo de desarrollo comercial, lo cual se traduzca en la presencia de marcas internacionales o en la existencia de establecimientos 
comerciales con formatos modernos. De hecho, hasta la actualidad el comercio local minorista, tanto de productos perecederos como de prendas textiles o artefactos electrónicos, se concentra en tiendas independientes de pequeñas dimensiones o en los mercados de abastos (INEI, 2015a).

En Cusco, como en otras ciudades del interior del Perú, el desembarco del retail moderno se ha producido de una manera más lenta que en las ciudades costeras y que en la capital, Lima. Sirva de ejemplo que el único centro comercial que existe en la ciudad imperial se inauguró recién en junio de 2014.

Como es fácil deducir, el contacto del consumidor cusqueño con un formato comercial como el que se analiza en este artículo ha sido muy limitado o inexistente. En ese contexto, esta investigación se planteó dos objetivos concretos: a) conocer qué factores de atracción de un centro comercial son los más valorados por estos nuevos usuarios. Ya se puede presuponer que éstos pueden ser diferentes a otros consumidores con una larga experiencia en la compra en este formato comercial. b) Proponer una segmentación de estos nuevos consumidores que atienda a variables demográficas (como la edad, el género o el nivel educativo) y a los factores de atracción que resultaron identificados como los más valorados (González-Hernández y Orozco-Gómez, 2012), pero que también incorpore variables como el conocimiento de marcas, frecuencia de visitas y permanencia en el centro comercial, las cuales hemos valorado que están asociadas al éxito de un centro comercial.

En suma, este trabajo resulta especialmente relevante porque aborda el estudio de los consumidores cusqueños, quienes no han tenido contacto previo con el formato de un centro comercial, y también por el contexto económico y demográfico propio de la ciudad de Cusco. En ese sentido, cabe destacar que entre 2008 y 2013, gracias al fuerte crecimiento de las inversiones mineras y de hidrocarburos realizadas en la zona, Cusco fue la región que más creció en todo el mundo, lo que se tradujo en el incremento del poder adquisitivo de sus pobladores (Instituto Peruano de Economía, 2013). En lo demográfico, la ciudad está sufriendo una fuerte transformación que se debería traducir en una fuente de innumerables oportunidades para el retail moderno. Según los últimos datos demográficos publicados, la población de Cusco alcanzó las 450000 personas en 2015, de las cuales 63.3\% tiene menos de 35 años, con una estimación de crecimiento de alrededor de 38\% en los próximos siete años (INEI, 2015b). En ese escenario, el artículo centró su objeto de estudio en los consumidores con un rango de edad de 25 a 35 años, quienes representan el grupo más importante de potenciales usuarios del centro comercial, pues los consumidores jóvenes han sido identificados como aquellos que sienten una mayor atracción hacia tales establecimientos y que están más predispuestos a visitarlos (Chan Yin-Fah et al., 2012).

\section{Revisión De Literatura}

A lo largo del tiempo, el concepto de un centro comercial o mall y su estudio ha ido evolucionado. En la actualidad, los centros comerciales son definidos como un grupo de establecimientos que son planificados, desarrollados, pertenecen y son administrados como propiedad única (International Council of Shopping Centers, 2008).

El estudio de los centros comeciales y de sus factores de atracción tiene como punto de partida el trabajo realizado por Huff (1963) al que habría que añadir, como referencia en este tipo de análisis, las investigaciones realizadas con posterioridad por autores como Lindquist (1974), Bearden y Teel (1983), Howard (1997) o Ruiz (1999). Desde estas primeras aproximaciones, el concepto de este tipo de superficie, así como el perfil de los consumidores que concurren en él ha ido cambiando, de modo que Kotler (2003) afirmaba que el consumidor se había vuelto más sofisticado y que el servicio se había convertido en una parte relevante para la adquisición de un producto.

En el Perú, a diferencia de lo que sucede en países como España, donde la aparición de los primeros centros comerciales se remonta a 1980 (AECC, 2008), estos establecimientos han surgido y se han popularizado en los últimos diez años gracias a la fuerte inversión nacional y extranjera en el sector, que alcanzó la cifra récord de 507 millones de dólares de inversión directa en 2013. De acuerdo con datos recientes, en 2014 en el país existía un total de 71 establecimientos como el estudiado (ACCEP, 2014). 
Una de las principales razones para explicar este boom inversor en centros comerciales a lo largo del país es la buena aceptación que este tipo de establecimiento ha conseguido (Regalado et al., 2009). En el Perú se está confirmando, como ha quedado evidenciado en otros países de Latinoamérica (D’Andrea $e t$ al., 2010), que dichos establecimientos ejercen gran poder de atracción sobre los consumidores (Ammani, 2013); Frantal et al., 2012) debido a la cantidad de tiendas, ofertas y servicios poco comunes que ofrecen y que hasta hace pocos años no existían en el país (Calvo y Calvo, 2013), lo que ha motivado que algunos autores les atribuyan, incluso, la capacidad de conseguir cambiar los hábitos de consumo locales (Rivas y Esteban, 2010).

Desde un punto de vista teórico, y como un primer objetivo del trabajo, se realizó una revisión de los resultados de estudios previos llevados a cabo en diferentes países europeos, asiáticos y africanos que han contribuido a definir factores de atracción cada vez más completos para identificar los atributos que los componen y adaptarlos a las realidades de cada país, región o ciudad. En Latinoamérica los estudios sobre los factores de atracción son escasos y se centran básicamente en las investigaciones ejecutadas por De Salles Vance et al. (2009) y Cavalcante y Campos (2013) en Brasil y por González-Hernández y Orozco-Gómez (2012) en México. En el caso concreto de Perú no hay un estudio sobre factores de atracción a centros comerciales centrados en el consumidor. Aunque de modo indirecto, aporta en ello la investigación de Regalado et al. (2009) que se decantó por identificar los factores asociados al éxito económico de los malls en la ciudad de Lima. Al respecto, determina esta relación por la ubicación (radio de influencia, segmentación de mercado, área disponible), las vías de acceso que ofrece (vías principales e ingresos, medios de transportes, accesos peatonales), el tenant mix, la estimación correcta de la demanda, la administración centralizada del establecimiento, el diseño, infraestructura, tamaño y dimensiones del centro comercial, la oferta de créditos al consumo y, por último, a la existencia de tiendas anclas.

La tabla 1 recoge los atributos más valorados en las investigaciones posteriores al 2000 que han resultado pertinentes, los cuales están agrupados en cinco factores de atracción hacia los centros comerciales: a) ubicación, b) tenant mix, c) popularidad, $d$ ) instalaciones y $e$ ) incentivos. Estos cinco factores fueron definidos originalmente por Wong et al. (2001), quienes valoraron y agruparon 21 atributos mediante un análisis factorial. A pesar de que en las investigaciones referidas el número y nombre de los atributos y factores analizados varía, en la mayoría de los casos es posible agruparlos siguiendo el patrón de cinco factores antedicho que permite ordenar de una forma sencilla todos los posibles atributos a valorar sin descuidar una visión integral de las características de un centro comercial.

TABLA 1

Investigaciones de factores de atracción hacia centros comerciales-atributos más valorados

\begin{tabular}{|c|c|c|c|c|c|c|c|}
\hline Año & País & Autor(es) & $\begin{array}{l}\text { Grupo } 1 \text { - } \\
\text { Ubicación }\end{array}$ & $\begin{array}{c}\text { Grupo } 2 \text { - } \\
\text { Calidad }\end{array}$ & $\begin{array}{c}\text { Grupo } 3 \text { - } \\
\text { Popularidad }\end{array}$ & $\begin{array}{c}\text { Grupo } 4 \text { - } \\
\text { Instalaciones }\end{array}$ & $\begin{array}{l}\text { Grupo } 5 \text { - } \\
\text { Incentivos }\end{array}$ \\
\hline 2004 & Sudáfrica & North y Kotze & & $\begin{array}{l}\text { Variedad de } \\
\text { tiendas } \\
\text { Variedad de } \\
\text { servicios }\end{array}$ & & & $\begin{array}{l}\text { Ampliación } \\
\text { de horarios } \\
\text { Entreteni- } \\
\text { miento }\end{array}$ \\
\hline 2005 & Inglaterra & Dennis & Accesibilidad & $\begin{array}{l}\text { Variedad de } \\
\text { tiendas }\end{array}$ & $\begin{array}{l}\text { Calidad de las } \\
\text { tiendas }\end{array}$ & $\begin{array}{l}\text { Layout } \\
\text { Ambientación } \\
\text { Limpieza }\end{array}$ & \\
\hline 2006 & España & $\begin{array}{l}\text { Munuera } \\
\text { Alemán y } \\
\text { Cuestas Díaz }\end{array}$ & & $\begin{array}{l}\text { Variedad de } \\
\text { tiendas }\end{array}$ & & Ambientación & Oferta de ocio \\
\hline
\end{tabular}


Tabla 1 (continúa)

\begin{tabular}{|c|c|c|c|c|c|c|c|}
\hline 2006 & $\begin{array}{l}\text { Emiratos } \\
\text { Árabes } \\
\text { Unidos }\end{array}$ & El-Adly & Convenience & $\begin{array}{l}\text { Variedad de } \\
\text { tiendas } \\
\text { Variedad de } \\
\text { servicios }\end{array}$ & Lujo & $\begin{array}{l}\text { Confort, mall } \\
\text { essence }\end{array}$ & $\begin{array}{l}\text { Ofertas de } \\
\text { ocio }\end{array}$ \\
\hline 2008 & Austria & $\begin{array}{l}\text { Teller y } \\
\text { Reutterer }\end{array}$ & Tenat Mix & $\begin{array}{l}\text { Merchandise } \\
\text { value }\end{array}$ & Especialización & & \\
\hline 2008 & Brasil & $\begin{array}{l}\text { De Salles Vance, } \\
\text { Felisoni de } \\
\text { Angelo y Dias } \\
\text { Fouto }\end{array}$ & & $\begin{array}{l}\text { Variedad de } \\
\text { tiendas }\end{array}$ & & Seguridad & $\begin{array}{l}\text { Oferta de ocio } \\
\text { Patio de } \\
\text { comidas }\end{array}$ \\
\hline 2009 & Eslovenia & Cirman y Pahor & Accesibilidad & $\begin{array}{l}\text { Variedad de } \\
\text { tiendas } \\
\text { Variedad de } \\
\text { servicios }\end{array}$ & $\begin{array}{l}\text { Calidad de las } \\
\text { tiendas } \\
\text { Calidad de los } \\
\text { servicios }\end{array}$ & $\begin{array}{l}\text { Parking } \\
\text { Limpieza }\end{array}$ & \\
\hline 2013 & Brasil & $\begin{array}{l}\text { Cavalcante y } \\
\text { Campos }\end{array}$ & $\begin{array}{l}\text { Localización } \\
\text { Acesibilidad }\end{array}$ & $\begin{array}{l}\text { Variedad de } \\
\text { tiendas }\end{array}$ & & $\begin{array}{l}\text { Servicios } \\
\text { generales } \\
\text { Transporte } \\
\text { interno } \\
\text { Climatización } \\
\text { Seguridad }\end{array}$ & $\begin{array}{l}\text { Oferta de ocio } \\
\text { Patio de } \\
\text { comidas } \\
\text { Cine } \\
\text { Tiendas ancla } \\
\text { Restaurantes }\end{array}$ \\
\hline 2011 & Irán & Abghari & & & & Ambientación & \\
\hline 2012 & Arabia Saudí & Saad Alqahtani & Cercanía & $\begin{array}{l}\text { Variedad de } \\
\text { productos }\end{array}$ & & $\begin{array}{l}\text { Limpieza } \\
\text { Climatización }\end{array}$ & Uso familiar \\
\hline 2012 & Ghana & $\begin{array}{l}\text { Anning-Dorson, } \\
\text { Kastner y } \\
\text { Mahmoud }\end{array}$ & & & & $\begin{array}{l}\text { Seguridad } \\
\text { Layout } \\
\text { Diseño }\end{array}$ & $\begin{array}{l}\text { Ofertas de } \\
\text { ocio }\end{array}$ \\
\hline 2012 & México & $\begin{array}{l}\text { González- } \\
\text { Hernández y } \\
\text { Orozco-Gómez }\end{array}$ & & & $\begin{array}{l}\text { Calidad de los } \\
\text { servicios }\end{array}$ & $\begin{array}{l}\text { Ambientación } \\
\text { Layout }\end{array}$ & \\
\hline 2013 & España & Calvo y Calvo & & $\begin{array}{l}\text { Variedad de } \\
\text { productos }\end{array}$ & & & $\begin{array}{l}\text { Ofertas de } \\
\text { ocio }\end{array}$ \\
\hline 2013 & Lituania & $\begin{array}{l}\text { Gudonaviciene y } \\
\text { Alijosiene }\end{array}$ & Cercanía & & $\begin{array}{l}\text { Calidad de las } \\
\text { tiendas }\end{array}$ & & $\begin{array}{l}\text { Patio de } \\
\text { comidas } \\
\text { Restaurantes }\end{array}$ \\
\hline 2014 & Polonia & $\begin{array}{l}\text { Mikołajczyk, } \\
\text { Borusiak y } \\
\text { Pierański }\end{array}$ & $\begin{array}{l}\text { Localización } \\
\text { Accesibilidad }\end{array}$ & $\begin{array}{l}\text { Variedad de } \\
\text { productos } \\
\text { Variedad de } \\
\text { servicios }\end{array}$ & $\begin{array}{l}\text { Calidad de los } \\
\text { servicios }\end{array}$ & $\begin{array}{l}\text { Ambientación } \\
\text { Layout }\end{array}$ & $\begin{array}{l}\text { Horario } \\
\text { extendido }\end{array}$ \\
\hline 2015 & Malasia & $\begin{array}{l}\text { Ab Majid, } \\
\text { Zalika Adnan, } \\
\text { Said y Olapin }\end{array}$ & & & & $\begin{array}{l}\text { Ambientación } \\
\text { Layout }\end{array}$ & $\begin{array}{l}\text { Programas de } \\
\text { fidelización }\end{array}$ \\
\hline 2014 & Sudáfrica & $\begin{array}{l}\text { Dubihlela y } \\
\text { Dubihlela }\end{array}$ & Accesibilidad & $\begin{array}{l}\text { Variedad de } \\
\text { productos }\end{array}$ & $\begin{array}{l}\text { Variedad de } \\
\text { marcas }\end{array}$ & $\begin{array}{l}\text { Ambientación } \\
\text { Parking }\end{array}$ & \\
\hline 2015 & India & $\begin{array}{l}\text { Sharma y } \\
\text { Chadha }\end{array}$ & Convenience & $\begin{array}{l}\text { Variedad de } \\
\text { tiendas }\end{array}$ & $\begin{array}{l}\text { Popularidad } \\
\text { Imagen pública }\end{array}$ & Ambientación & $\begin{array}{l}\text { Programas de } \\
\text { fidelización } \\
\text { Promociones } \\
\text { Ofertas de } \\
\text { ocio }\end{array}$ \\
\hline
\end{tabular}

Fuente: elaboración propia. 
Como se aprecia en la tabla 1, el factor tenat mix resulta ser el más valorado en casi todas las investigaciones, y sus atributos más destacados son la variedad de tiendas y la variedad de servicios y de productos. Al mismo tiempo, se observa que los factores incentivos e instalaciones son juzgados como relevantes en casi todos los casos, pero sus atributos más importantes varían por país. Así, en el caso de los incentivos, la presencia de la oferta de ocio y la existencia de patios de comida es transversal a todos los estudios, a lo que hay que añadir que North y Kotze (2004) y Mikołajczyk et al. (2014) identificaron la importancia de la amplitud de horarios, y que Ab Majid et al. (2015) y Sharma y Chadha (2015) destacaron la relevancia de los programas de fidelización. En el caso de las instalaciones, la ambientación y el layout fueron atributos identificados como destacados por casi todos los autores. Sin embargo, la seguridad, en Brasil y Ghana (De Salles Vance et al. 2009; Cavalcante y Campos, 2013; Anning-Dorson et al., 2012) surgió como un atributo muy demandado por los usuarios. La climatización también fue un atributo valorado por Cavalcante y Campos (2013) y Saad Alqahtani (2012).

En cuanto al factor popularidad, éste tiene como atributo más importante la calidad de los servicios y de las tiendas. Al mismo tiempo, El-Adly (2006) enumeró que el lujo era significativo, mientras que la especialización del local fue identificada por Teller y Reutterer (2008). Por su parte, Gudonaviciene y Alijosiene (2013) y Sharma y Chadha (2015) hallaron que la popularidad, la marca y la imagen del establecimiento eran transcendentales para los usuarios.

En el caso del factor ubicación, si bien no todos los autores creen que es determinante para que los usuarios se inclinen por la elección de un centro comercial, el atributo nombrado de manera más recurrente por los investigadores es la accesibilidad, a lo que habría que añadir la localización tal y como indican Cavalcante y Campos (2013) y Mikołajczyk et al. (2014) o la cercanía como afirman Saad Alqahtani (2012) y Gudonaviciene y Alijosiene (2013).

Como se aprecia en el análisis previo, casi todos los factores de atracción identificados están compuestos por atributos de carácter racional, pero como indicaron North y Kotze (2004) y Cadogan et al. (2007) los centros comerciales se han convertido también para muchas personas en opciones atractivas de ocio y, por tanto, en generadores de experiencias y emociones. Esta línea de pensamiento ha sido reforzada por Chandrasekhar et al. (2013), quienes afirman que los malls son vistos como originadores o fuentes de estatus y reconocimiento social mediante el brand equity de la marca del centro comercial, su imagen y su prestigio, tal y como indican Bahar Isin et al. (2011), Gudonaviciene y Alijosiene (2013) y Sharma y Chadha (2015). Otra forma de generar emociones positivas es también a través de las marcas de los establecimientos adscritos a éste (Dubihlela y Dubihlela, 2014; Jiwaji, 2014; Rasa, 2013). En el caso particular de Perú, Arellano (2012) también sostiene que los factores emocionales son decisivos en el proceso de elección del centro comercial.

Como se ha mencionado, el segundo objetivo está centrado en crear un perfil detallado del nuevo usuario cusqueño de centros comerciales. Dicha segmentación aparece como necesaria, pues si bien los malls ejercen una fuerte atracción en cualquier país o ciudad donde se instalan, sus promotores deben adaptar su oferta a los estilos de vida de las personas que pueden acudir (Du Preez et al., 2007), así como también a las necesidades que tengan (D’Andrea et al., 2010; Chauhan et al., 2011), ya que de lo contrario se puede incurrir en errores que han sido reportados en la experiencia comparada cuando la oferta de un centro comercial no está alineada con el perfil del consumidor local (Choi et al., 2009).

En este punto, el estudio de las investigaciones previas permitió identificar que en la mayoría de los casos los perfiles de los usuarios de centros comerciales se construyen sólo con variables demográficas. Así, por ejemplo, Gilboa (2009), De Salles Vance et al. (2009) o Brantley et al., (2011) usaron la edad para corroborar que ésta condicionaba la preferencia hacia la oferta de servicios de un centro comercial. Del mismo modo, el género fue usado por Kuruvilla y Ranjan (2008) con igual propósito. Por último, se encontraron estudios previos que confirmaron que el nivel formativo de los usuarios influye en la percepción positiva o negativa hacia los centros comerciales y, en consecuencia, la valoración de los factores de atracción también varía (Allard et al., 2009). 
A la fecha, no existen investigaciones concretas sobre el usuario de centros comerciales en ciudades del interior del Perú, a pesar de que ostentan las mejores tasas de crecimiento económico de los últimos años (Guevara, 2012). Las únicas investigaciones existentes se han enfocado en el perfil general del consumidor de las provincias peruanas. Así, los estudios de mercado disponibles se enfocan en las ciudades de Piura y Trujillo, ubicadas en la costa norte del país (Wakabayashi, 2014); en Cajamarca y Huancayo, ciudades de la sierra norte y central del país respectivamente (Wakabayashi, 2014); en la ciudad de Pucallpa, de la región selva (Wakabayashi, 2014) y en la ciudad de Arequipa, ubicada en la sierra sur del país (Arellano, 2012).

Todos estos estudios tienen como característica común resaltar que el consumidor de dichas regiones valora el reconocimiento social que se otorga al hecho de comprar nuevas marcas y productos inexistentes hasta hace poco en dichos lugares, lo que se asocia a estatus o a éxito personal (Chaparro, 2013). Asimismo, dichas investigaciones dan cuenta de que el consumidor provinciano promedio se define como un trabajador esforzado, gracias a lo cual ha alcanzado un alto poder adquisitivo que le permite comprar un gran número de productos inimaginables para ellos hasta hace pocos años (Wakabayashi, 2014). Respecto a la percepción del retail moderno, el poblador del interior del país suele relacionarlo con un mayor estándar de calidad de vida, con la modernidad, la aparición de nuevas marcas y el surgimiento de nuevas formas de pago, principalmente con tarjetas de crédito (Chaparro, 2013).

\section{Metodología}

Este artículo estudió el comportamiento de los consumidores cusqueños en el único centro comercial de la ciudad que fue abierto recientemente. El estudio se dividió en dos fases: una exploratoria y otra descriptiva. Debido a la falta de información previa sobre la realidad del usuario cusqueño, sus propias peculiaridades y al hecho de que se pretendía estudiar su comportamiento frente a un fenómeno nuevo en la ciudad, en una primera fase se ejecutaron dos técnicas de investigación cualitativa: dos entrevistas en profundidad a expertos locales en retail y comportamiento del consumidor y 20 entrevistas en profundidad a usuarios del centro comercial que cumplían las características del público objetivo analizado con el fin de ver en qué medida los resultados de las investigaciones previas eran adaptables al contexto local (Patton, 1980). En el caso de las entrevistas a expertos, se aplicaron a un investigador de mercados cusqueño y al administrador del nuevo centro comercial. Estas investigaciones usaron un cuestionario estructurado de preguntas abiertas cuya finalidad fue definir qué atributos, de todos los propuestos en los resultados de las investigaciones que realizaron los autores consultados en el marco teórico (tabla 1), podían servir para identificar, en una segunda fase de carácter cuantitativa, los factores de atracción que se ajustaban a la realidad cusqueña. En el caso de los expertos, se mencionan algunas de las frases más destacadas de sus reflexiones: "El consumidor cusqueño es exigente y espera encontrar buenos productos siempre" o "en la actualidad el cusqueño es como un bebé en el mundo del retail moderno, las variables que actualmente valora cuando va al nuevo mall son limitadas y no se pueden comparar con otras realidades" y "el mall es la nueva atracción de la ciudad con nuevos servicios nunca antes vistos como cines, patios de comida o tiendas por departamentos". Por el lado de los usuarios, se encuentran las siguientes: "Me encanta encontrar siempre ofertas" o "el centro comercial es el lugar donde me encuentro con mis amigos y me gusta que sea moderno" y "es increíble la cantidad de cosas nuevas que hay en el mall". Fruto del estudio de estas investigaciones preliminares se determinó que los 15 atributos recogidos en la tabla 2 debían ser evaluados en la segunda fase, ya que tanto los expertos como los usuarios los repitieron frecuentemente.

En la segunda fase, através de un muestreo probabilístico simple, se llevó a cabo una investigación cuantitativa a una muestra de 378 personas de Cusco con edades comprendidas entre los 25 y 35 años a quienes se les sometió a un cuestionario, el cual respondieron de manera voluntaria. La información fue recogida de manera presencial en el centro comercial mediante una encuesta estructurada de 31 preguntas con opciones simples y múltiples, así como también con escalas de Likert. El cuestionario estuvo dividido en tres bloques. El primero se centró en 
variables demográficas tales como la edad, el género, el lugar de residencia y el nivel educativo. En el segundo, se usaron escalas de Likert con valores comprendidos del 1 al 5 para que los usuarios valoraran la importancia de cada uno de los 15 atributos identificados en la investigación cualitativa (tabla 2). El último bloque incluyó preguntas sobre frecuencia de visitas, razones para acudir, personas con las que acuden, tiempo de permanencia en el local y conocimiento de las marcas presentes en el establecimiento. La muestra estuvo conformada por 378 individuos, divididos en hombres y mujeres de entre 25 y 35 años que asisten al único centro comercial en la ciudad. Como resultado del trabajo de campo, la muestra estuvo compuesta como se expone en el cuadro 1.

TABLA 2

Resultados investigación cualitativa-atributos valorados

\begin{tabular}{|lllll}
\hline $\begin{array}{l}\text { Variedad de produc- } \\
\text { tos y servicios }\end{array}$ & $\begin{array}{c}\text { Ofertas o promocio- } \\
\text { nes }\end{array}$ & \multicolumn{1}{c}{ Seguridad } & Oferta de cines & Amplitud de horarios \\
\hline Amplitud de horarios & Patio de comidas & $\begin{array}{l}\text { Calidad de los pro- } \\
\text { ductos y servicios }\end{array}$ & Servicios posventa & $\begin{array}{l}\text { Existencia de nuevas } \\
\text { marcas }\end{array}$ \\
Atención personal & $\begin{array}{l}\text { Reconocimiento } \\
\text { social }\end{array}$ & $\begin{array}{l}\text { Experiencia de } \\
\text { compra }\end{array}$ & Comodidad & Cercanía \\
\hline
\end{tabular}

Fuente: elaboración propia.

CUADRO 1

Composición de la muestra

\begin{tabular}{|c|c|c|c|c|c|}
\hline Edad & 25 a 28 años & 29 a 32 años & \multicolumn{2}{|c|}{33 a 35 años } & Total \\
\hline Frecuencia & 211 & 106 & \multicolumn{2}{|c|}{61} & 378 \\
\hline Porcentaje & $55.8 \%$ & $28.0 \%$ & \multicolumn{2}{|c|}{$16.1 \%$} & $100 \%$ \\
\hline Género & \multicolumn{2}{|c|}{ Masculino } & \multicolumn{2}{|c|}{ Femenino } & Total \\
\hline Frecuencia & \multicolumn{2}{|c|}{184} & \multicolumn{2}{|c|}{194} & 378 \\
\hline Porcentaje & \multicolumn{2}{|c|}{$48.7 \%$} & \multicolumn{2}{|c|}{$51.3 \%$} & $100 \%$ \\
\hline Estudios & Sin estudios & Secundaria & Posgrado & Instituto/ Universitario & Total \\
\hline Frecuencia & 6 & 64 & 63 & 245 & 378 \\
\hline Porcentaje & $1.6 \%$ & $16.9 \%$ & $16.7 \%$ & $64.8 \%$ & $100 \%$ \\
\hline
\end{tabular}

Fuente: elaboración propia.

Además, los resultados del trabajo de campo fueron codificados y posteriormente sometidos a un análisis bivariado y factorial con rotación varimax tal y como Henry et al. (2005) y Shaked (2009) recomiendan en sus estudios o como lo hizo González-Hernández y Orozco-Gómez (2012) en su investigación para identificar los factores de atracción del consumidor mexicano. Posteriormente, para definir los segmentos del consumidor cusqueño se aplicó un análisis de cluster jerárquico con un método aglomerativo (Henry et al., 2005; Shaked, 2009) debido a que el punto de partida inicial era una muestra reducida de la que no se conocía de antemano el número de clusters existentes (Visauta, 2002).

\section{Resultados}

Fruto del análisis factorial al que se sometieron los 15 atributos que fueron valorados por los consumidores cusqueños se pudo identificar cuatro factores que explican $72.6 \%$ de las variaciones. Con la finalidad de validar el instrumento, se sometió a una prueba de consistencia interna mediante la prueba del coeficiente de Alfa de Cronbach. Los atributos y los factores fueron evaluados conjunta e individualmente (cuadro 3). Los resultados de dicha prueba se encontraron siempre entre 0.62 y 0.91 que, por ejemplo para esta investigación, son valores con- 
siderados aceptables o muy buenos (George y Mallery, 2003). Además la prueba de la media Kaiser-Meyer-Olkin arrojó un resultado de 0.86 , lo que corrobora que la muestra es adecuada. En consecuencia, los cuatro factores que aparecen juzgados como relevantes por los consumidores cusqueños de acuerdo con esta investigación fueron identificados como $a$ ) servicios comunes, $b$ ) anclas, $c$ ) calidad y $d$ ) reconocimiento.

El primer factor (servicios comunes) está integrado por atributos como la amplitud de horarios, variedad de productos y servicios, la existencia de ofertas o promociones, la cercanía y la comodidad. Este factor es el de mayor importancia y explica por sí solo $41.1 \%$ de la variación y, tal como refleja su nombre, está estrechamente relacionado con los servicios básicos que ofrece un centro comercial.

El segundo factor (anclas) está conformado por atributos como la seguridad, el patio de comidas, la oferta de cines, el servicio posventa y la existencia de nuevas marcas. Este segundo factor refleja la importancia que tiene para los asistentes al centro comercial la presencia de diferentes marcas, así como de establecimientos anclas.

Referente al tercer factor (calidad), éste incorpora los atributos de la calidad de los productos y servicios y la atención personalizada, todos ellos centrados en el valor añadido de los servicios o productos. Por último, el cuarto factor (reconocimiento) está compuesto por los atributos vinculados al reconocimiento social y a la experiencia de compra dentro del centro comercial. De los cuatros factores relevantes, éste es el único que está relacionado con atributos emocionales, mientras que los tres que le preceden tienen una clara orientación racional (cuadro 2 y cuadro 3 ).

Ahora bien, para conseguir una adecuada segmentación de los consumidores cusqueños se clasificaron los resultados de la investigación mediante clusters jerárquicos en función de los cuatro factores de atracción identificados en la fase previa. Como resultado de este análisis, se puedo establecer la existencia de dos grupos diferenciados de consumidores. El primer grupo compuesto por $88.4 \%$ de la muestra analizada y el segundo representado por $11.6 \%$ (cuadro 4 y cuadro 5).

\section{CUADRO 2}

Matriz de componente rotado

\begin{tabular}{lcccc}
\hline & \multicolumn{3}{c}{ Componente } \\
\cline { 2 - 4 } & 1 & 2 & 3 & 4 \\
\hline Variedad & 0.771 & 0.116 & 0.230 & 0.344 \\
Reconocimiento & 0.279 & 0.153 & 0.070 & 0.840 \\
Ofertas & 0.756 & 0.171 & 0.199 & 0.270 \\
Comodidad & 0.601 & 0.589 & 0.153 & 0.053 \\
Cercanía & 0.742 & 0.385 & 0.134 & -0.143 \\
Seguridad & 0.364 & 0.767 & -0.011 & -0.003 \\
Horarios & 0.863 & 0.159 & 0.222 & 0.152 \\
Patio de comidas & 0.231 & 0.728 & 0.014 & 0.355 \\
Cine & 0.296 & 0.680 & 0.164 & 0.235 \\
Experiencia de compra & 0.052 & 0.242 & 0.571 & 0.578 \\
Atención & 0.195 & 0.286 & 0.705 & 0.075 \\
Posventa & -0.015 & 0.642 & 0.542 & -0.051 \\
Nuevas marcas & 0.003 & 0.621 & 0.512 & 0.122 \\
Calidad servicio & 0.281 & 0.039 & 0.819 & 0.108 \\
Calidad producto & 0.235 & -0.009 & 0.820 & 0.058 \\
\hline
\end{tabular}




\section{CUADRO 3}

Extracción de factores-análisis Alfa de Cronbach

\begin{tabular}{|llcc}
\hline \multirow{3}{*}{ Factores } & \multicolumn{1}{c}{ Variables } & \multicolumn{2}{c}{ Componente } \\
\cline { 3 - 4 } Servicios comunes & Horarios & Extracción & Alfa de Cronbach \\
& Variedad & 0.863 & \\
& Ofertas & 0.771 & 0.892 \\
& Cercanía & 0.756 & \\
& Comodidad & 0.742 & \\
& Seguridad & 0.601 & 0.827 \\
Anclas & Patio de comidas & 0.767 & \\
& Cine & 0.728 & \multirow{2}{*}{0.811} \\
& Posventa & 0.680 & \\
Calidad & Nuevas marcas & 0.642 & \multirow{2}{*}{0.628} \\
& Calidad Producto & 0.621 & \\
Reconocimiento & Calidad Servicio & 0.820 & \\
& Atención & 0.819 & \\
& Reconocimiento & 0.705 & 0.840 \\
\end{tabular}

Fuente: elaboración propia.

CUADRO 4

Resumen de los resultados del análisis cluster-segmentos de consumidores

\begin{tabular}{|llcc}
\hline & & Frecuencia & Porcentaje \\
\hline \multirow{3}{*}{ Válido } & Optimistas & 334 & $88.4 \%$ \\
& Pesimistas & 44 & $11.6 \%$ \\
& Total & 378 & $100 \%$ \\
\hline
\end{tabular}

Fuente: elaboración propia.

CUADRO 5

Resumen de los resultados del análisis cluster-segmentos de consumidores-factores-análisis Anova

\begin{tabular}{lccccc}
\hline Cluster-Factores & Servicios básicos & Anclas & Calidad & Reconocimiento \\
\hline Optimistas & 4.27 & 3.56 & 3.92 & 3.52 \\
Pesimistas & Media & 2.40 & 2.16 & 2.97 & 2.07 \\
Total & 4.05 & 3.40 & 3.81 & 3.35 \\
\hline Sig. & 0.00 & 0.00 & 0.00 & 0.00 \\
\hline
\end{tabular}

Fuente: elaboración propia. 
Con el fin de poder construir un perfil más detallado del usuario del nuevo centro comercial cusqueño, una vez identificados los dos grupos de usuarios que valoran de manera diferenciada los factores de atracción, se procedió a crear sus perfiles evaluando también sus rasgos sociodemográficos, la relación con el centro comercial y con los establecimientos que ahí están ubicados.

Los nuevos segmentos de consumidores se validaron mediante el uso de las pruebas de chi cuadrado y Anova (cuadro 6 y cuadro 7). Las variables que se evaluaron fueron edad, género, lugar de residencia (cuadro 8) y nivel de estudios, razones para visitar el centro comercial, acompañantes con los que acuden, frecuencia de la visita, tiempo de permanencia en el centro comercial y marcas que conoce presentes en el establecimiento. Los dos grupos identificados fueron denominados como los Entusiastas y los Pesimistas. La descripción de cada uno de ellos se detalla en los cuadros 6 y 7.

\section{CUADRO 6}

Resumen de los resultados del análisis cluster-segmentos de consumidores-perfiles-chi cuadrado

\begin{tabular}{|c|c|c|c|c|c|}
\hline & & \multicolumn{2}{|c|}{ Cluster-Factores } & \multirow{2}{*}{ Total } & \multirow{2}{*}{ Sig. } \\
\hline & & Optimistas & Pesimistas & & \\
\hline \multirow{3}{*}{ Edad } & $25-28$ & $52.7 \%$ & $79.5 \%$ & $55.8 \%$ & \multirow{3}{*}{0.001} \\
\hline & $29-32$ & $29.0 \%$ & $20.5 \%$ & $28.0 \%$ & \\
\hline & $33-35$ & $18.3 \%$ & $0.0 \%$ & $16.1 \%$ & \\
\hline \multirow{2}{*}{ Género } & Masculino & $48.2 \%$ & $52.3 \%$ & $48.7 \%$ & \multirow{2}{*}{0.612} \\
\hline & Femenino & $51.8 \%$ & $47.7 \%$ & $51.3 \%$ & \\
\hline \multirow{8}{*}{ Distrito } & Ccorca & $0.0 \%$ & $6.8 \%$ & $0.8 \%$ & \multirow{8}{*}{0.000} \\
\hline & Cusco & $28.1 \%$ & $52.3 \%$ & $31.0 \%$ & \\
\hline & Poroy & $3.6 \%$ & $6.8 \%$ & $4.0 \%$ & \\
\hline & San Jerónimo & $18.3 \%$ & $6.8 \%$ & $16.9 \%$ & \\
\hline & San Sebastián & $6.3 \%$ & $13.6 \%$ & $7.1 \%$ & \\
\hline & Santiago & $10.2 \%$ & $0.0 \%$ & $9.0 \%$ & \\
\hline & Saylla & $5.4 \%$ & $6.8 \%$ & $5.6 \%$ & \\
\hline & Wanchaq & $28.1 \%$ & $6.8 \%$ & $25.7 \%$ & \\
\hline \multirow{4}{*}{ Estudios } & Posgrado & $1.8 \%$ & $0.0 \%$ & $1.6 \%$ & \multirow{4}{*}{0.006} \\
\hline & Superior & $15.6 \%$ & $27.3 \%$ & $16.9 \%$ & \\
\hline & Secundaria & $15.0 \%$ & $29.5 \%$ & $16.7 \%$ & \\
\hline & Sin estudios & $67.7 \%$ & $43.2 \%$ & $64.8 \%$ & \\
\hline \multirow{5}{*}{$\begin{array}{l}\text { Frecuencia de } \\
\text { visita }\end{array}$} & Diario & $6.0 \%$ & $6.8 \%$ & $6.1 \%$ & \multirow{5}{*}{0.00} \\
\hline & Interdiario & $10.2 \%$ & $0.0 \%$ & $9.0 \%$ & \\
\hline & Semanal & $53.6 \%$ & $50.0 \%$ & $53.2 \%$ & \\
\hline & Dos semanas & $22.2 \%$ & $13.6 \%$ & $21.2 \%$ & \\
\hline & Mensual & $8.1 \%$ & $29.5 \%$ & $10.6 \%$ & \\
\hline \multirow{4}{*}{ Conoce marcas } & Todas & $21.3 \%$ & $22.7 \%$ & $21.4 \%$ & \multirow{4}{*}{0.170} \\
\hline & Unas pocas & $64.4 \%$ & $56.8 \%$ & $63.5 \%$ & \\
\hline & Casi ninguna & $10.8 \%$ & $20.5 \%$ & $11.9 \%$ & \\
\hline & Ninguna & $3.6 \%$ & $0.0 \%$ & $3.2 \%$ & \\
\hline
\end{tabular}


CUADRO 6 (continúa)

\begin{tabular}{|llrrrr}
\hline \multirow{5}{*}{ Tiempo de perma- } & $1-2 \mathrm{~h}$ & $18.9 \%$ & $29.5 \%$ & $20.1 \%$ & \\
nencia & $2-4 \mathrm{~h}$ & $59.0 \%$ & $50.0 \%$ & $57.9 \%$ & \\
& $4-6 \mathrm{~h}$ & $19.2 \%$ & $6.8 \%$ & $17.7 \%$ & 0.001 \\
& $>6$ & $0.9 \%$ & $6.8 \%$ & $1.6 \%$ & \\
& Otro & $2.1 \%$ & $6.8 \%$ & $2.6 \%$ & \\
\multirow{3}{*}{ Acompañante } & Familia & $31.4 \%$ & $48.9 \%$ & $50.8 \%$ & \\
& Amigos & $33.8 \%$ & $12.8 \%$ & $49.7 \%$ & 0.000 \\
& Pareja & $25.4 \%$ & $6.4 \%$ & $37.0 \%$ & \\
& Solo & $8.2 \%$ & $19.1 \%$ & $14.0 \%$ & \\
& Otros & $1.3 \%$ & $12.8 \%$ & $3.4 \%$ & \\
\hline
\end{tabular}

Fuente: elaboración propia.

CUADRO 7

Resumen de los resultados del análisis cluster-segmentos de consumidores-perfiles Anova

\begin{tabular}{lcccc}
\hline Cluster & \multicolumn{3}{c}{ Media } & Sig. \\
\cline { 2 - 4 } Factores & Optimistas & Pesimistas & Total & 0.000 \\
\hline $\begin{array}{l}\text { Encuentro } \\
\text { Familia } \\
\text { Visita }\end{array}$ & 3.41 & 2.75 & 3.33 & 0.001 \\
Ropa & 3.69 & 3.18 & 3.63 & 0.029 \\
$\begin{array}{l}\text { Visita } \\
\text { Alimentos }\end{array}$ & 3.22 & 2.80 & 3.17 & 0.000 \\
$\begin{array}{l}\text { Visita } \\
\text { Pasear }\end{array}$ & 3.59 & 2.52 & 3.46 & 0.000 \\
$\begin{array}{l}\text { Visita } \\
\text { Comer }\end{array}$ & 3.33 & 2.70 & 3.26 & 0.007 \\
$\begin{array}{l}\text { Visita } \\
\text { Cine } \\
\text { Encuentro } \\
\text { Amigos }\end{array}$ & 3.45 & 2.98 & 3.39 & 0.000 \\
\hline
\end{tabular}

Fuente: elaboración propia.

\section{CUADRO 8}

Distritos de Cusco y distancia al centro comercial

\begin{tabular}{lc}
\hline Distrito & Distancia \\
\hline Wanchaq & $2.3 \mathrm{~km}$ \\
Santiago & $4.8 \mathrm{~km}$ \\
San Jerónimo & $9.8 \mathrm{~km}$ \\
Cusco & $3.8 \mathrm{~km}$ \\
San Sebastián & $2.9 \mathrm{~km}$ \\
Saylla & $18.1 \mathrm{~km}$ \\
Poroy & $12.5 \mathrm{~km}$ \\
Ccorca & $26.4 \mathrm{~km}$ \\
\hline
\end{tabular}

Fuente: elaboración propia. 
a) Perfil 1-Los Optimistas: es el segmento más grande, ya que está integrado por $88.4 \%$ de los consumidores cusqueños que acuden al centro comercial. En términos generales, valoran positivamente los cuatro factores de atracción, pero le dan especial importancia al factor servicios comunes (media de 4.27 sobre 5). Este grupo está formado indistintamente por hombres o mujeres de cualquier edad que viven en los distritos aledaños al centro comercial, como Wanchap (28.1\%) o el centro histórico (28.1\%), y en su mayoría carece de algún grado formativo (67.7\%). En cuanto a su relación con el centro comercial, los Optimistas acuden acompañados casi siempre de alguien (90.5\%), de su familia (31.4\%), pareja (25.4\%) o amigos (25.4\%). Por otro lado, argumentan que la razón principal por la que visitan el centro comercial es para comprar ropa (3.69 de media sobre 5), pero en segundo y tercer lugar afirman que van simplemente para encontrarse con amigos (3.65 de media sobre 5) o a pasear (3.59 de media sobre 5). En referencia a la frecuencia con la que asisten al centro comercial, $53.6 \%$ acude de manera semanal en tanto que $22.2 \%$ lo hace de modo bimensual. También hay un número significativo de personas que asiste de manera diaria o interdiaria (16.2\%) llegando a permanecer en el establecimiento la mayoría de las veces entre 2 y 4 horas (59\%) y en algunos casos entre 4 y 6 horas (19.2\%). De todas maneras, aunque acuden con tanta frecuencia, es paradójico que estos consumidores afirmen que conocen pocas o casi ninguna de las marcas del centro comercial (75.2\%).

b) Perfil 2-Los Pesimistas: representan $11.6 \%$ del conjunto de la muestra y valoran de manera negativa todos los factores de atracción. En este grupo el género es irrelevante, pero no así la edad y el grado de estudios alcanzado, ya que este perfil está formado principalmente por los consumidores más jóvenes (79.5\%) y, al contrario de los Optimistas, la mayoría cuenta con algún grado formativo (56.8\%). Además, se aprecia que, si bien una parte importante de estos usuarios vive en el centro de la ciudad (52.3\%), es también representativo (20.5\%) el porcentaje que vive en los distritos más alejados como Ccorca, Poroy o Saylla. Por otro lado, el usuario con este perfil se relaciona de una manera diferente con el centro comercial, pues si bien acude con su familia (48.9\%), también lo suele hacer solo (19.1\%). A ello habría que añadir que aunque la frecuencia de visita más repetida es semanal (50\%), un número importante acude de manera bimensual (13.6\%) o mensual (29.5\%). Los periodos de permanencia son menores a cuatro horas (79.5\%) y el motivo por el que concurren al mall es exclusivamente a comprar ropa (3.18 de media sobre 5); afirman que no van a encontrarse con amigos, pasear, ir al cine o a comer al food court. Al igual que en el caso de los Optimistas, los Pesimistas no conocen las marcas que están en el centro comercial (77.3\%).

\section{Conclusiones}

Como fruto del análisis de los resultados, se puede afirmar que el perfil de los consumidores cusqueños debido a su falta de experiencia previa con este formato comercial estudiado comparte algunos rasgos comunes con otros consumidores citados en algunas investigaciones previas, pero al mismo tiempo es destacable que hay factores que hasta al momento no son relevantes a pesar de que se citan de manera repetitiva en muchos de esos estudios previos. En particular, en este trabajo los factores de atracción determinantes para los consumidores cusqueños son los servicios comunes, primer factor, y la calidad, tercer factor, debido a que seguramente perciben que los productos y servicios que ofrece el centro comercial son de mayor calidad y más modernos (Cirman y Pahor, 2009). Estos resultados permiten identificar que los cusqueños comparten algunas de las creencias sobre atributos básicos de un centro comercial que fueron hallados en investigaciones previas en el extranjero y que concluyeron que la amplitud de horarios (North y Kotze, 2004; Mikołajczyk et al. (2014) y la variedad de productos y servicios (North y Kotze, 2004; El-Adly, 2006; Cirman y Pahor, 2009; Saad Alqahtani, 2012; Calvo y Calvo, 2013; Mikołajczyk et al., 2014; Dubihlela y Dubihlela, 2014), ambos atributos incluidos en el primer factor, así como la calidad, tercer factor (Cirman y Pahor, 2009; Cavalcante y Campos, 2013; Gudonaviciene y Alijosiene, 2013), eran decisivos en la atracción de consumidores hacia los centros comerciales. 
Sin embargo, cuando se valoran factores aspiracionales o emocionales, esta investigación descubrió que no son relevantes para los cusqueños para determinar su preferencia por los centros comerciales, a diferencia de lo sostenido por North y Kotze (2004), Cadogan et al. (2007), Chandrasekhar et al. (2013) y en el Perú por Arellano (2014), ya que el cuarto factor, reconocimiento, fue el menos valorado. Esto puede estar relacionado con una escasa implementación de estrategias de branding por parte de las empresas globales presentes en el centro comercial, las cuales resultan importantes tal y como plantean Dubihlela y Dubihlela (2014), Rasa (2013) y Jiwaji, (2014). Una segunda razón puede deberse al bajo brand equity de la marca del mall y que según las investigaciones de Bahar Isin et al. (2011), Gudonaviciene y Alijosiene (2013) y Sharma y Chadha (2015) es determinante para la elección de un centro comercial. Además, como se describe en los resultados, los dos perfiles de usuarios cusqueños desconocen las marcas y, en el caso de los Pesimistas, se llegan a mostrar reacios y críticos hacia el propio centro comercial y las marcas que ahí se encuentran. Esta afirmación es especialmente significativa en el caso de las personas más jóvenes y con algún nivel formativo. Tales datos contradicen los proporcionados por Chan Yin-Fah et al. (2012) a nivel internacional, quienes hallaron que los consumidores jóvenes y los usuarios de las provincias, respectivamente, se sentían atraídos y preferían las nuevas marcas presentes en los centros comerciales. En el caso concreto del Cusco se identifica que las marcas en general están asociadas a productos de calidad, pero aparentemente no son adquiridas por un tema aspiracional o de reconocimiento social.

Por otro lado, si consideramos que las personas sin grados formativos son aquellas que disponen de menores rentas, este trabajo confirma el hallazgo realizado por Allard et al. (2009), quienes concluyeron que los consumidores de menores rentas mostraban mayor interés por los centros comerciales y sus ofertas que los otros segmentos de consumidores.

Por el contrario, el segundo factor (anclas) es poco valorado por el público objetivo y refuta los resultados de investigación realizada en Sudamérica por De Salles Vance et al. (2009) o Cavalcante y Campos (2013) según la cuales la seguridad o las marcas eran atributos valorados por los consumidores. Seguramente, esto pueda obedecer a que el contexto cusqueño dista del de las otras ciudades sudamericanas estudiadas por De Salles Vance $e t$ al. (2009) o Cavalcante y Campos (2013), en primer lugar, debido a que los problemas de seguridad en Cusco no son tan graves como en las otras ciudades de América del Sur analizadas en dichos estudios y, en segundo lugar, a que se han estudiado a consumidores con un mayor contacto y experiencia con otros formatos de tiendas modernas, lo cual se ha evidenciado a lo largo de este trabajo y no pasa con el consumidor cusqueño, quien recién en 2014 ha entrado en contacto por vez primera con un formato moderno de retail.

Dentro del mismo segundo factor, los patios de comida o la existencia de cines son también atributos poco apreciados por los consumidores cusqueños, lo que no se alinea con los hallazgos de De Salles Vance et al. (2009), Cavalcante y Campos (2013), Gudonaviciene y Alijosiene (2013), quienes indicaban que tales atributos resultaban de suma importancia para la elección de un centro comercial. En esta investigación, este resultado se refuerza cuando los entrevistados fueron interrogados sobre las razones por las que acuden al centro comercial, ya que ir a comer logró una puntuación de 3.26 sobre 5, una media muy inferior comparada con las obtenidas por las otras razones expuestas que señalan que van a pasear, a encontrarse con amigos o comprar ropa. Tales datos evidencian que hasta el momento los cusqueños identifican al centro comercial sólo como punto de encuentro y como un lugar donde la oferta de prendas para vestir es más amplia. Aparentemente, se podría afirmar que el consumidor todavía está explorando el nuevo establecimiento, le llama la atención, pero aún no entiende toda la oferta comercial y servicios que ofrece el mall. En concreto, y a pesar de que las tiendas ancla de comida deberían funcionar como generadores de tráfico dentro del mall (Haseki, 2013; Hattingh et al. (2014), en el caso particular del Cusco la investigación revela que no son servicios valorados al momento de decidir si se visita o no el centro comercial. Asimismo, vale la pena resaltar que acudir al mall para comprar comida es la razón menos llamativa para los usuarios, por lo que se podría afirmar que el supermercado no ejerce una función de tienda ancla. Quizás la razón principal de dichos hallazgos se deba a que el tipo de comida que ofrece el centro comercial cusqueño está poco adaptada a los gustos y costumbres culinarias propias de la zona, donde la adecuación de la oferta a la 
realidad local es un elemento decisivo para el éxito del centro comercial tal y como afirman Du Preez et al. (2007), D’Andrea et al. (2010) o Chauhan et al. (2011).

Desde el punto de vista gerencial, los resultados son de gran utilidad tanto para los administradores del centro comercial como para quienes lo son de las marcas adscritas al lugar. Como ha sido explicado, el estudio de los factores por los cuales los consumidores cusqueños son atraídos hacia el centro comercial ha permitido identificar la existencia de dos perfiles diferenciados de consumidor con percepciones antagónicas del mall y sus atributos, por lo que sería conveniente plantear objetivos y estrategias de marketing diferenciadas para cada uno de ellos.

Los usuarios Positivos tienen una percepción positiva de los servicios comunes como un factor importante para visitar o comprar en el centro comercial, pero, al mismo tiempo, el reconocimiento o las anclas son factores de atracción que tienen poca relevancia para este segmento. Por tanto, definir objetivos y estrategias de marketing destinados a ampliar las razones por las cuales estos usuarios acuden al centro comercial sería oportuno. Fruto de una correcta implementación de dichas estrategias, se lograría que los usuarios fueran no sólo a pasear o a encontrarse con amigos sino también para ir al cine o para el uso de los servicios de restauración. Además, sería conveniente adaptar la oferta de supermercados o patios de comidas a los gustos y costumbres locales, ya que en la actualidad dicha oferta está estandarizada. También, resultaría deseable plantear estrategias de comunicación con el fin de dar a conocer las beneficios y educar en el uso de los servicios que ofrece el centro comercial.

Para los Pesimistas el mall en su conjunto es percibido como algo negativo, por lo que sería acertado plantear estrategias destinadas a mejorar la imagen del centro comercial, ya que de lo contrario se podría correr el riesgo de que se deteriore aún más sobre todo si se toma en cuenta que este grupo está formado por un importante número de jóvenes y que este segmento crecerá de manera considerable en los próximos años según indica el INEI.

Finalmente, es importante resaltar que el hecho de que los usuarios cusqueños no hayan tenido contacto previo con otros formatos comerciales modernos permite entender por qué tanto la marca centro comercial como las marcas adscritas a éste son poco o nada conocidas por $75.4 \%$ de los usuarios. Ante esta realidad, los administradores de estas empresas se encuentran ante el reto de adaptar su oferta comercial al ciclo de apredizaje de los cusqueños, en la cual es una opción posible la implementación estrategias de branding que contribuyan a lograr posicionar sus marcas y que se vuelvan relevantes (Dubihlela y Dubihlela, 2014; Rasa, 2013; Jiwaji, 2014) en un mercado virgen como es en la actualidad el Cusco. Sin duda, en la medida que estas estrategias de branding de las empresas presentes en el mall se implementen y se adapten con éxito será probable que los usuarios del establecimiento pasen de valorar sólo factores básicos, como los identificados en el estudio del nuevo centro comercial, a apreciar el valor emocional y experencial que puede proporcionarles.

\section{Prospectiva}

Este artículo ha permitido, por primera vez en Latinoamérica, estudiar cuáles son los factores de atracción hacia un centro comercial en un mercado particular, como es el cusqueño, el cual está conformado por usuarios que no han tenido contacto previo con este formato de retail moderno. Se definieron cuatro factores de atracción, aunque el número de atributos evaluados para su construcción ha sido menor al de otras investigaciones debido al desconocimiento y falta de experiencia de los usuarios estudiados con este formato comercial. De este modo, sería relevante analizar con una investigación de carácter transversal si el número de atributos se incrementa con el paso del tiempo, así como si la composición y complejidad de los factores se ven modificadas.

Asimismo, si bien los perfiles creados se adaptan a la realidad cusqueña en comparación a los que se identificaron en los trabajos consultados, sería muy útil poder completarlos con otros usuarios dentro del mismo país o región e incorporar a la investigación atributos de carácter psicológico, sociológico y etnográfico que puedan permitir conocerlos a mayor profundidad.

Por último, la limitación que se presenta se debe a que la investigación se centró en Cusco, por lo que resulta imposible corroborar si los resultados obtenidos son extrapolables a otras ciudades de la región o son un caso aisla- 
do. De cualquier manera, Cusco no es la única ciudad del Perú o de Latinoamérica donde los centros comerciales aparecen por primera vez como una opción comercial para muchos ciudadanos. Sería importante comparar estos resultados con los que se obtengan en ciudades que tengan similares características a las de Cusco.

\section{ReFEREnCIAS}

Abghari, M. (2011). Investigation of the effects of stores' tenant mix and internal and external environmental conditions on customer satisfaction from shopping centers in Iran. International Journal of Marketing Studies, 3(4), 158-164.

Ab Majid, R., Zalika Adnan, N., Said, N., \& Olaopin, O. (2015). Drivers influencing customers' visit to shopping centres in Malaysia. International Surveying Research Journal, 5(2), 43-58.

Allard, T., Babin, B. J., \& Chebat, J. C. (2009). When income matters: Customers evaluation of shopping malls: hedonic and utilitarian orientations. Journal of Retailing and Consumer Services, 16(1), 40-49.

Ammani, P. (2013). A study of the factors that influence customer preference for shopping malls over local markets. The IUP Journal of Management Research, 12(1), 7-21.

Anning-Dorson, T., Kastner, A., \& Abdulai, M. (2012). Investigation into mall visitation motivation and demographic idiosyncrasies in Ghana. Management Science Letters, 3(2), 38.

Arellano, O. (2012). Retail peruano. Centros comerciales y tiendas de ropa. Lima: Arellano Marketing.

Arellano, O. (2014). Encontrando oportunidades en el mercado de centros comerciales. Lima: Arellano Marketing.

Asociación de Centros Comerciales del Perú (ACCEP). (2014). Los centros comerciales en el Perú.

Asociación Española de Centros y Parques Comerciales (AECC). (2008). Directorio 2008 de los centros comerciales de España 2008. Asociación Española de Centros Comerciales, Madrid

Bahar Isin, F., Hakan Altintas, M., Kaufmann, H. R., \& Kilic, S. (2011). Mall equity measurement based on brand equity conceptualization. Journal of Business \& Retail Management Research, 5(2), 55-70.

Brantley, A., Jackson, V., \& Stoel, L. (2011). Mall attributes and shopping value: Differences by gender and generational cohort. Journal of Retailing and Consumer Services, 18(1), 1-9.

Bearden, W. P., \& Teel, J. E. (1983). Selected determinants of consumer satisfaction and complaint reports. Journal of Marketing Research, 20, 21-8

Cadogan, J. W., Farrel, A. M., Hart, C., Reed, G., \& Stachow, G. (2007). Enjoyment of the shopping experience: Impact on customers repatronage intentions and gender influence. The Service Industries Journal, 27(5), 583-604.

Calvo, C. y Calvo, D. (2013). Estudio exploratorio sobre los factores de atracción de centros comerciales: una aproximación al consumidor español. CIENCIA ergo-sum, 20(2), 107-120.

Cavalcante, F. e Campos, D. F. (2013). Satisfação e atractividade: como o público jovem avalia os shopping centers. RaUnP-Revista Electrónica do Mestrado Profissional em Administração da Universidade Potiguar, 5(2), 79-97

Cirman, A., \& Pahor, M. (2009). Sustaining the challenge from the outskirts: City centre retail viability in Ljubljana, Slovenia. Economic and business review, 11(3), 217-231.

Chan Yin-Fah, B., Jamaluddin, A., Osman, S., \& Wong, Y. T. (2012). Shopping motives, store attributes and shopping enjoyment among Malaysian youth. Journal of Retailing and Consumer Services, 19(2), 240-248.

Chandrasekhar, U., Mansurali, A., \& Swamynathan, R. (2013). Mall mania: A study of factors influencing consumers' preference towards shopping malls in Coimbatore City. The IUP Journal of Marketing Management, 12(4), 29-41. 
Chaparro, H. (2013). El retail y la cultura de consumo en el interior. Presentación. No disponible.

Chauhan, H., Gupta, R., \& Setia, P. (2011). Modern retailing in India: A study of the attractiveness of a shopping mall. Asia Pacific Journal of Research in Business Management, 2(12), 1-2.

Choi, A., Chan, G., \& Yannis K. (2009). Success and failure in Southern China shopping mall development. The Retail Digest, 16-21.

D’Andrea, G., Marcotte, D., \& Morrison, G. D. (2010). Let emerging market customers be your teachers. Harvard Business Review, 88(12), 115-120

Dennis, C. (2005). Objects of desire: consumer behaviour in shopping centre choices. Palgrave Macmillan.

De Salles Vance, P., Felisoni de Angelo, C., \& Martins Dias Fouto, N. (2009). Estudo do comportamento de compra do frequentador de shopping centers na cidade de São Paulo. ReA UFSM Revista de administração, 2(1),130-146.

Dubihlela, D., \& Dubihlela, J. (2014). Attributes of shopping mall image, customer satisfaction and mall patronage for selected shopping malls in Southern Gauteng, South Africa. Journal of Economics and Behavioral Studies, 6(8), 682-689.

Du Preez, R., Visser, E. M., \& Zietsman, L. (2007). Lifestyle, shopping orientation, patronage behaviour and shopping mall behaviour-A study of South African male apparel consumers. European Advances in Consumer Research, 8, 279-280.

El-Adly, M. I. (2006). Shopping malls attractiveness: A segmentation approach. International Journal of Retail \& Distribution Management, 35(11), 936-950.

Frantal, B., Kunc, J., Szczyrba, Z., \& Tonev, P. (2012). Shopping centres and selected aspects of shopping behaviour. Geographia Technica, 2, 39-51.

George, D., \& Mallery, P. (2003). SPSS for windows step by step: A simple guide and reference. 11.0. Update (4th ed.). Boston: Allyn \& Bacon.

Gilboa, S. (2009). A segmentation study of Israeli mall customers. Journal of Retailing and Consumer Services, 16(2), 135-144.

González-Hernández, E. M., \& Orozco-Gómez, M. (2012). A segmentation study of Mexican consumers based on shopping centre attractiveness. International Journal of Retail \& Distribution Management, 40(10), 759-777

Gudonaviciene, R., \& Alijosiene, S. (2013). Influence of shopping centre image attributes on customer choices. Economics \& Management, 18(3), 545-552.

Guevara, R. (2012). Crecimiento sostenido, competitividad y desarrollo económico regional del Perú. Revista Strategia Centrum Católica, 7(27), 40-44.

Haseki, M. I. (2013). Customer expectations in-mall restaurants: A case study. International Journal of Business and Society, 14(1), 41-60

Hattingh, Z., Mhlanga, O., \& Moolman, H. J. (2014). Expectations and perceptions of customers in-mall restaurants in an East London shopping mall using the DINESERV approach. African Journal for Physical, Health Education, Recreation and Dance. 20, 914-927.

Henry, D. B., Tolan, P. H., \& Gorman-Smith, D. (2005). Cluster analysis in family psychology research.Journal of Family Psychology, 19(1), 121-132

Howard, E. (1997). The Management of shopping centres: Conflict or collaboration. International Review of Retail, Distribution and Consumer Research, 7(3).

Huff, D. L. (1963). A probabilistic analysis of shopping center trade areas. Land Economics, 39(1), 81-90.

International Council of Shopping Centers (ICSC). (2008). Shopping Center Definitions. Disponible en https://www.icsc.org/news-and-views/research/shopping-center-definitions

Instituto Nacional de Estadísticas e Informática (INEI). (2015a). Establecimientos censados por actividad económica. Disponible en http://censos.inei.gob.pe/cenec2008/tabulados/\# 
Instituto Nacional de Estadísticas e Informática (INEI). (2015b). Población total al 30 de junio, por grupos quinquenales de edad, según departamento, provincia y distrito. Disponible en https://www.inei.gob. pe/estadisticas/indice-tematico/poblacion-y-vivienda/

Instituto Peruano de Economía (IPE). (2013). Foro CUSCO 2014-Logros y retos del desarrollo económico y social de Cusco. Disponible en http://www.ipe.org.pe/portal/wp-content/uploads/2018/02/Logros-y-Retos-del-Desarrollo-Econ\%C3\%B3mico-y-Social-de-Cusco-2014-Miguel-Palomino.pdf

Jiwaji, A. (2014). Brand fever hits Kenya. African Business, 407, 82-84.

Kotler, P. (2003). Marketing Management (11th ed). Prentice Hall International Editions.

Kuruvilla, S. J., \& Ranjan, K. (2008). Gender and mall shopping: An analysis of patronage patterns, shopping orientation and consumption of fashion of Indian youth. International Journal of Business Insights \& Transformation, 1(2), 1-8.

Lindquist, J. D. (1974). Meaning of image: survey of empirical and hypothetical evidence. Journal of Retailing, 50(4), 29-38.

Mikołajczyk, J., Borusiak, B., \& Pierański, B. (2014). Assessment of the attractiveness of shopping centres in Poland. 13th International Conference Marketing Trends Proceedings-Wenecja. ESCP-AEP European School of Management.

Munuera Alemán, J. L. y Cuestas Díaz, P. J. (2006). Factores de atracción de los centros comerciales de España. España: ICE.

North, E. J., \& Kotze, T. (2004). Customers perception of the attractiveness of shopping centres in Pretoria. Southern African Business Review, 8(1), 30-38.

Patton, M. Q. (1980). Qualitative evaluation methods. Beverly Hills.

Rasa, S. (2013). Influence of shopping centre image attributes on customer choices. Lithuania: Economics and Management, 18(3), 545-551.

Regalado, O., Fuentes, C., Aguirre, G., García, N., Román, M. y Vallejo, R. (2009). Factores criticos de éxito en los centros comerciales de Lima Metropolitana y el Callao. Lima: Universidad ESAN.

Rivas, J. A. y Esteban, I. G. (2010). Comportamiento del consumidor. Decisiones y estrategia de marketing (sexta edición).

Ruiz, F. J. (1999). Image of suburban shopping malls and two-stage versus uni-equational modelling of the retail trade attraction: an empirical application. European Journal of Marketing, 33(5,6),

Saad Alqahtani, S. (2012). Shopping centers attraction: Saudi customers perspective. Asia-Pacific Business Research Conference. Kuala Lumpur, Malaysia.

Shaked, G. (2009). A segmentation study of Israeli mall customers. Journal of Retailing and Consumer Services, $16,135-144$

Sharma, S., \& Chadha, S. K. (2015). Why do people shop at malls? A study on mall attractiveness factors of consumers of tier - ii cities. International Journal of Retailing \& Rural Business Perspectives, 4(2), 1578-1583.

Teller, C., \& Reutterer, T. (2008). The evolving concept of retail attractiveness: what makes retail agglomerations attractive when customers shop at them? Journal of Retailing and Consumer Services, 15(3), 127-143.

Visauta, B. (2002). Técnicas de análisis multivariante para investigación social y comercial. Madrid: Ra-Ma.

Wakabayashi, J. L. (2014). Impacto del crecimiento del retail en los hábitos de consumo del interior del país. Disponible en http://gestion.pe/economia/crece-canal-moderno-provincias-conoce-como-se-percibe-este-consumidor-2116395/1.

Wong, K. M., Lu, Y., \& Yuan, L. L. (2001). Scattr: An instrument for measuring shopping centre attractiveness. International Journal of Retail and Distribution Management, 29(2).

CC BY-NC-N 